\title{
Perspectiva masculina quanto a métodos contraceptivos
}

\author{
Male perspectives on contraceptive methods
}

\footnotetext{
1 Centro de Pesquisas das Doenças Materno-Infantis de Campinas. C. P. 6181, Campinas, $S P$ 13081-970, Brasil.
}

\begin{abstract}
Historically, family planning programs have targeted women almost exclusively. More recently it has become clear that men have a strong influence on their partners' use of contraception, either by opposing the use of any or one specific method, or by deciding that a woman has to either use a given method or be sterilized. In this context, our study aimed to evaluate the male perspective on the role of gender in the decision to use contraceptive methods and to have and raise children. The sample consisted of 776 males from a university in the State of São Paulo: 467 graduate students, 314 employees, and 129 teachers. A structured, previously tested questionnaire was used for the interviews. Data analysis is currently under way. The least familiar contraceptive methods were Billings (29.9\%), injectables (35.4\%), and jellies (57.8\%). Some 80\% of the interviewees mentioned other methods. The proportion of men who denied knowledge of any contraceptive method whatsoever was 1.6\%. Our findings are similar to those of other authors. Key words Contraception; Family Planning; Birth Control; Contraceptives Devices
\end{abstract}

Resumo Ao longo dos anos, os programas de planejamento familiar preocuparam-se quase que exclusivamente em atingir apenas a população feminina. Mais recentemente essa atitude vem mudando, pois evidenciou-se que os homens têm forte influência sobre suas parceiras quanto ao uso de anticoncepcionais. Dentro deste contexto, a presente pesquisa teve como objetivo geral identificar a perspectiva e o papel masculino quanto à decisão de usar métodos contraceptivos. O tamanho amostral foi calculado em 776 homens de uma universidade paulista; foram selecionados 467 estudantes de graduação, 314 funcionários e 129 docentes, totalizando 910 sujeitos. Foi utilizado um questionário estruturado e pré-testado. A análise dos dados encontra-se em andamento. Os métodos menos conhecidos pelos homens foram: Billings (29,9\%), injetáveis (35,4\%) e os cremes/geléias (57,8\%). Os demais foram mencionados por pelo menos $80 \%$ dos entrevistados. A proporção dos que disseram não conhecer qualquer meio de evitar a gravidez esteve em torno de 1,6\%. Estes resultados são semelhantes aos encontrados por outros autores.

Palavras-chave Anticoncepção; Planejamento Familiar; Controle da Natalidade; Dispositivos Anticoncepcionais 


\section{Introdução}

O planejamento familiar é uma atividade que diz respeito não somente ao casal que o pratica, pois tem implicações sócio-demográficas. Dentro da área médica, é uma das mais importantes atividades preventivas, sendo seu objetivo principal proporcionar aos casais e, em particular, às mulheres as informações e os meios necessários para que possam decidir o número de filhos que desejam e quando querem tê-los, de forma consciente e voluntária. Planejar o tamanho da família, o número de filhos desejados e o espaçamento entre eles são direitos de cada indivíduo (Pinto, 1987). No Brasil, este direito é garantido pela Constituição (CN, 1988).

Apesar de ser um direito de homens e mulheres, durante muitos anos, os programas de planejamento familiar esqueceram-se que os homens também deveriam participar das decisões reprodutivas. Pressupunha-se que estes tivessem pouco interesse pelo assunto e, conseqüentemente, pouco se pesquisou até hoje sobre o papel e a atitude masculina em face desta questão. Uma possível explicação para isto é que a regulação da fecundidade era e é feita, na grande maioria dos casos, pelas mulheres, uma vez que é a mulher que engravida, sofre as conseqüências e riscos da gestação e assume maior responsabilidade pelo cuidado dos filhos. Assim, a maior parte dos métodos contraceptivos está dirigido para o público feminino e somente o condom, a vasectomia e o coito interrompido para os homens. Portanto, as mulheres são o público preferencial desses programas (Population Reports, 1987).

Esta conduta começou a mudar a partir da década de 80, quando esses programas passaram a buscar estratégias que permitissem alcançar um número maior de usuários; foi nesse momento que a saúde reprodutiva masculina começou a receber alguma atenção (Rogow, 1992). Evidenciou-se que, independente de ser o homem ou a mulher que faça uso de algum método contraceptivo, o homem tem um papel, tanto na escolha, quanto no uso do método (Hardy, 1971; Population Reports, 1987; Faúndes et al., 1989).

Alguns autores já têm pesquisado a participação masculina na anticoncepção. Na cidade de São Paulo, mais de $80 \%$ dos homens entrevistados, que viviam em união, conheciam ou tinham ouvido falar de: condom, pílula, laqueadura, tabela e coito interrompido (Sakamoto et al., 1989). Resultados semelhantes quanto a conhecimento do condom foram relatados por Berquó \& Souza (1991). As autoras salientam, entretanto, que, embora a pergunta tivesse sido feita visando explicitamente ao que o homem poderia fazer, muitos se referiram ao uso de contraceptivos pelas mulheres.

Pelos resultados das pesquisas já realizadas nesta área, verifica-se que os homens têm um papel na escolha do método contraceptivo a ser usado pelo casal e, quando perguntados sobre o assunto, evidenciam um conhecimento superficial. Além disso, observa-se que existem poucos dados disponíveis acerca da atitude masculina quanto à anticoncepção, conhecimento e uso de métodos anticoncepcionais. No presente, em que se enfatiza tanto a necessidade de os homens assumirem suas responsabilidades reprodutivas, fica mais evidente essa lacuna de conhecimento.

Por outro lado, um dos assuntos de mais destaque nas recentes conferências sobre população e desenvolvimento (Cairo em 1994) e sobre a mulher (Beijing em 1995) foi a questão da responsabilidade masculina quanto à reprodução.

Nesse contexto de reivindicações quanto à participação masculina nas questões de saúde reprodutiva, este projeto de pesquisa teve como objetivo investigar o conhecimento dos homens e suas opiniões sobre os métodos contraceptivos, percepção acerca de sua participação e responsabilidade na prevenção da gravidez, das DST-AIDS, bem como sua percepção acerca dos diferentes papéis de gênero em relação às decisões sobre quando ter relação sexual e uma gravidez.

\section{Sujeitos e método}

O tamanho amostral, tendo como referência o total de homens de uma universidade paulista, foi calculado em 776. Este cálculo foi baseado em uma proporção de $1 \%$ de desconhecimento de métodos contraceptivos, estimado na população (Diaz, 1994), com uma diferença de 0,7 pontos percentuais $(70 \%)$ entre a proporção populacional e amostral e com intervalo de confiança de $95 \%$. Foram selecionados, com partilha proporcional, 467 estudantes de graduação, 314 funcionários e 129 docentes, totalizando 910 homens. Esta seleção foi realizada de forma sistemática por meio de três listas: uma com os nomes dos docentes, outra com os nomes dos funcionários e a terceira com os nomes dos alunos. A cada sete homens selecionou-se um, sendo que, para o início do processo, foi sorteado um entre os sete primeiros de cada lista. Se por algum motivo, após três tentativas, não fosse possível entrevistar o 
sujeito selecionado, o mesmo era substituído pelo seguinte da respectiva lista. Os dados foram coletados mediante entrevistas individuais, realizadas preferencialmente na universidade; quando isto não foi possível, foram agendadas para outro local. Um questionário estruturado, pré-testado, com perguntas précodificadas e textuais, foi utilizado nas entrevistas. O banco de dados que contém as perguntas pré-codificadas está sendo checado para possíveis inconsistências; as perguntas textuais encontram-se em fase de codificação. A seguir são apresentados alguns resultados já disponíveis, referentes ao conhecimento e uso de métodos anticoncepcionais e questões de gênero.

\section{Resultados parciais}

Mais da metade dos entrevistados tinha até trinta anos de idade por ocasião da entrevista e a grande maioria se autoclassificou como sendo branca a sua cor ou raça $(83,5 \%)$. Como era esperado, dada a constituição da amostra, três quartos dos entrevistados tinham grau de escolaridade superior, completo ou incompleto (Tabela 1).

Quanto a algumas decisões da vida familiar, possivelmente associadas à perspectiva de gênero, a grande maioria $(>90,0 \%)$ respondeu que o casal é o responsável por estas decisões. A responsabilidade de sustentar a família foi a que teve maior percentagem de resposta que atribuía ao homem esse dever (8\%), e a de evitar as gestações foi a que teve mais $(6,4 \%)$ respostas que a atribuía à mulher. Ninguém atribuiu somente ao homem a responsabilidade de cuidar dos filhos (Tabela 2).

A percentagem de entrevistados que referiu não conhecer qualquer um dos métodos contraceptivos foi de 1,6\%. Entre os que referiram conhecer pelo menos um anticoncepcional, os métodos menos referidos foram: Billings $(29,9 \%)$, injetáveis $(35,4 \%)$ e cremes/geléias $(57,8 \%)$. Os demais foram mencionados por pelo menos $80 \%$ dos entrevistados (Tabela 3$)$.

Entre os homens entrevistados que viviam em união conjugal ou que referiram ter uma parceira sexual fixa e que estavam usando algum método, a camisinha e a pílula eram os métodos contraceptivos mais usados $(33,4 \% \mathrm{e}$ $29,2 \%$, respectivamente). Ressalte-se que a laqueadura tubária foi o terceiro método mais citado $(20,8 \%)$ e a vasectomia teve uma percentagem de uso pouco inferior à tabela, 7,6\% e $9,0 \%$, respectivamente. Alguns homens referi-
Tabela 1

\begin{tabular}{|c|c|c|}
\hline & $\mathrm{n}$ & $\%$ \\
\hline Alunos & 467 & 51,3 \\
\hline Funcionários & 314 & 34,5 \\
\hline Docentes & 129 & 14,2 \\
\hline \multicolumn{3}{|l|}{ Idade } \\
\hline Até 19 & 121 & 13,3 \\
\hline $20-29$ & 378 & 41,5 \\
\hline $30-39$ & 185 & 20,3 \\
\hline $40-49$ & 135 & 14,8 \\
\hline $50-59$ & 62 & 6,8 \\
\hline 60 ou mais & 29 & 3,2 \\
\hline \multicolumn{3}{|l|}{ Cor* } \\
\hline Branca & 758 & 83,5 \\
\hline Negra/Parda & 84 & 9,2 \\
\hline Outros & 66 & 7,3 \\
\hline \multicolumn{3}{|l|}{ Escolaridade** } \\
\hline Primário & 80 & 8,8 \\
\hline Ginásio & 63 & 7,0 \\
\hline Colegial & 83 & 9,1 \\
\hline Superior & 677 & 74,4 \\
\hline
\end{tabular}

* Faltou informação de dois homens.

** Grau completo ou incompleto.

Tabela 2

Percepção masculina sobre quem deve decidir acerca de temas que envolvem questões de gênero $(n=910)$.

\begin{tabular}{|c|c|c|c|c|}
\hline Temas & o homem & a mulher & ambos & outros \\
\hline Sustentar a família* & 8,0 & 0,1 & 91,3 & 0,7 \\
\hline Decisões financeiras ${ }^{\star *}$ & 2,8 & 1,7 & 95,1 & 0,4 \\
\hline Cuidar dos filhos** & - & 4,7 & 95,0 & 0,2 \\
\hline Prevenção de gravidez* & 1,2 & 6,4 & 92,3 & 0,1 \\
\hline Quando ter relação sexual* & 1,1 & 1,5 & 97,0 & 0,4 \\
\hline
\end{tabular}

* Faltou informação de um homem.

** Faltou informação de dois homens.

ram o uso de uma combinação de métodos, tais como tabela e condom, ou até mesmo condom e pílula. Quando perguntados sobre quem escolheu o método usado pelo casal, pouco mais da metade dos entrevistados referiu ter sido uma decisão em conjunto (55,5\%). Pouco mais de um quinto, entretanto, referiu ter sido a parceira quem decidiu sobre o método a ser usado, e 13\% disseram que o médico é que havia decidido (Tabela 4 ). 
Tabela 3

Conhecimento de métodos contraceptivos* $(n=909)$.

\begin{tabular}{lcc}
\hline Métodos & $\mathbf{n}$ & $\%$ \\
\hline Condom & 894 & 98,2 \\
Pílula & 889 & 97,7 \\
Vasectomia & 875 & 96,1 \\
Laqueadura & 859 & 94,4 \\
Tabela & 841 & 92,4 \\
DIU & 838 & 92,1 \\
Coito interrompido & 792 & 87,0 \\
Diafragma & 730 & 80,2 \\
Geléia, creme vaginal & 526 & 57,8 \\
Injetavéis & 322 & 35,4 \\
Billings & 272 & 29,9 \\
Outros & 77 & 8,5 \\
Nenhum & & 1,6 \\
\hline
\end{tabular}

* Referidos espontaneamente ou por estimulação.

Tabela 4

Método contraceptivo em uso pelos casais* por ocasião da entrevista e pessoa que o escolheu $(n=476)$.

\begin{tabular}{|c|c|c|}
\hline Métodos ${ }^{\star \star}$ & $\mathrm{n}$ & $\%$ \\
\hline \multicolumn{3}{|c|}{ Com participação masculina } \\
\hline Condom & 159 & 33,4 \\
\hline Tabela & 43 & 9,0 \\
\hline Vasectomia & 36 & 7,6 \\
\hline Coito interrompido & 18 & 3,8 \\
\hline Billings & 1 & 0,2 \\
\hline \multicolumn{3}{|c|}{ Sem participação masculina } \\
\hline Pílula & 139 & 29,2 \\
\hline Laqueadura & 99 & 20,8 \\
\hline DIU & 32 & 6,7 \\
\hline Injetáveis & 6 & 1,3 \\
\hline Diafragma & 4 & 0,8 \\
\hline Geléia, creme vaginal & 3 & 0,6 \\
\hline Outros & 3 & 0,6 \\
\hline \multicolumn{3}{|l|}{ Pessoa que escolhe $e^{\star \star \star}$} \\
\hline O casal & 264 & 55,5 \\
\hline O homem & 40 & 8,4 \\
\hline A mulher & 106 & 22,3 \\
\hline O médico & 62 & 13,0 \\
\hline Outra pessoa & 1 & 0,2 \\
\hline
\end{tabular}

* Incluídos apenas homens que tinham parceiras e estavam usando algum método contraceptivo.

** Faltou informação de dois homens.

*** Faltou informação de um homem.

\section{Comentários}

A grande maioria dos homens entrevistados revelou conhecer diferentes métodos contraceptivos. A proporção dos que disseram não conhecer qualquer meio de evitar a gravidez foi semelhante à encontrada por Diaz (1994) entre estudantes universitários. Por outro lado, em relação ao conhecimento dos diferentes métodos anticoncepcionais, as proporções encontradas foram semelhantes às verificadas entre mulheres em pesquisas do mesmo tipo (Arruda et al., 1987; Bemfam \& Macro International, 1992; Osis et al., 1993).

Entretanto, entre os resultados já obtidos, foi surpreendente verificar a alta proporção de uso de métodos anticoncepcionais masculinos ou que implicam a participação masculina. Se considerarmos em conjunto o uso de condom, vasectomia, tabela, coito interrompido e Billings, observamos que pouco mais da metade (54\%) dos métodos em uso implicavam a maior ou menor participação do homem. Esse resultado contrapõe-se ao que rotineiramente se constata, que é a participação predominantemente feminina na anticoncepção (Arruda et al., 1987; Pinotti et al., 1990; Bemfam \& Macro International, 1992).

O que mais chama a atenção nos resultados é a atitude aparentemente pouco machista do grupo de homens estudado. A imensa maioria expressa um grande equilíbrio de gênero quando perguntada sobre decisões referentes à família, incluindo a vida sexual. É claro que a expressão de opinião equilibrada quanto à relação de gênero, durante uma entrevista, não é necessariamente um indicador fiel de qual seja a verdadeira conduta nesse grupo de homens. É possível que a resposta corresponda mais àquilo que consideraram correto responder, à conduta que deveria ser adotada, do que propriamente à forma de comportamento na vida diária.

Existe, por exemplo, uma grande diferença entre os $92 \%$ que dizem ser de responsabilidade do casal a prevenção da gravidez e os 55\% que referem ter sido o casal que efetivamente escolheu o método contraceptivo em uso. Entretanto, mesmo se os homens realmente desejassem participar nessa decisão, seria possível que uma parte de suas parceiras assumissem, independente deles, qual método usar para prevenir-se de uma gestação. Se quiséssemos ser otimistas quanto às relações de gênero dentro do grupo estudado, é possível interpretar que as diferenças entre esses $92 \%$ e $55 \%$ não correspondem necessariamente à falta de sinceridade na resposta à pergunta feita. De qualquer forma, essa aparente mudança favorável 
a um maior equilíbrio de gênero confirma-se nas condutas dos homens na prática da anticoncepção, que aparece compartilhada em partes quase iguais entre homens e mulheres. Isto seria o esperado se a responsabilidade de evitar a gestação fosse de ambos.

Portanto, apesar de surpreendente, fica difícil não interpretar os resultados como uma indicação inequívoca de mudança da atitude masculina quanto às relações de gênero na área da anticoncepção, no sentido de maior equilíbrio de poder, tão desejável, e que parecia estar tão longe de ser atingido.

É verdade, entretanto, que o grupo estudado representa, sem dúvida, uma elite muito particular, não só pelo alto nível de educação, mas por receber a influência de um ambiente universitário de vanguarda, onde boa parte dos indivíduos encontra-se exposta à ampla discussão dos problemas sociais do país, incluindo aqui as questões de gênero. Esta não é, evidentemente, a situação da grande maioria da população, que ainda apresenta em torno de

\section{Agradecimentos}

Aos funcionários do Cemicamp pela colaboração na realização do projeto e à Fundação Ford pelo financiamento.

\section{Referência}

ARRUDA, J. M.; RUTENBERG, N.; MORRIS, L. \& FERRAZ, E. A., 1987. Pesquisa Nacional sobre Saúde Materno-Infantil e Planejamento Familiar, Brasil, 1986. Rio de Janeiro: Sociedade Civil Bem-Estar Familiar no Brasil (Bemfam) \& Instituto para Desenvolvimento de Recursos.

BEMFAM (Sociedade Civil Bem-Estar Familiar no Brasil) \& MACRO INTERNATIONAL, 1992. Pesquisa sobre Saúde Familiar no Nordeste - Brasil. Relatório de Pesquisa. Rio de Janeiro: Bemfam \& Macro Internacional.
13\% de analfabetismo (IBGE, 1994) e um sistema de educação elementar altamente precário. Apesar disso, parece-nos que os resultados aqui relatados são extremamente alentadores, já que se sabe que muitas das mudanças culturais iniciam-se entre os indivíduos com maior nível de instrução e daí difundem-se para o resto da população (Coleman et al., 1964; Botomore, 1964; Heilborn, 1992).

Uma vez confirmadas essas observações, as mesmas viriam opor-se ao conceito tão difundido de que seria inútil desenvolver anticoncepcionais masculinos, visto que os homens não iriam usá-los. Os resultados sugerem, entretanto, que a responsabilidade reprodutiva está começando a ser assumida pelos homens, pelo menos nesses grupos cujas condutas tendem, supostamente, a ser imitadas pelo restante da sociedade.

Será muito importante comparar esses resultados com os de outros grupos e de outras regiões do Brasil, bem como finalizar a tarefa de analisá-los por completo.
BERQUÓ, E. \& SOUZA, M. R., 1991. Conhecimento e Uso do Condom. Anticoncepção e Prevenção de Doenças Sexualmente Transmissiveis. Textos Nepo no 20. Campinas: Núcleo de Estudos de População - Nepo/Unicamp.

BOTTOMORE, T. B., 1964. Elites and Society. New York: Penguin Books.

CN (Congresso Nacional), 1988. Constituição da República Federativa. São Paulo: Imprensa Oficial do Estado. 
COLEMAN, J.; MENZEL, H. \& KATZ, E., 1964. Social process in physicians adoption of a new drug. In: Social Change: Sources, Patterns and Consequences (A. Etzioni \& E. Etzione, eds.), pp. 440454. New York/London: Basic Books.

DIAZ, M., 1994. Condutas Sexuais e Atitudes que Constituem Fatores de Risco para Gravidez Não Desejada e AIDS em Estudantes Universitários: Um Desafio para a Educação Sexual. Dissertação de Mestrado, Campinas: Faculdade de Educação, Universidade Estadual de Campinas.

FAÚNDES, A.; HARDY, E. \& PINOTTI, J. A., 1989. Commentary on womens's reproductive health. Means or end? International Journal of Gynecology and Obstetrics, 3(suppl.):115-118.

HARDY, E., 1971. Influencia de algunas características del esposo sobre el uso de anticonceptivos por su mujer - estudio de parejas. Cuadernos Médicos Sociales, 1(35):34-41.

HEILBORN, M. L., 1992. Vida a dois: conjugalidade igualitária e identidade sexual. In: VIII Encontro Nacional de Estudos Populacionais. São Paulo: Associação Brasileira de Estudos Populacionais.

IBGE (Instituto Brasileiro de Geografia e Estatística), 1994. Anuário Estatístico do Brasil. Rio de Janeiro: IBGE.
OSIS, M. J.; HARDY, E.; SIMÕES, I. R. S.; VERA, S. \& FAÚNDES, A., 1993. Laqueadura tubária nos serviços de saúde do Estado de São Paulo. Revista de Ginecologia e Obstetrícia, 1:195-204.

PINOTTI, J. A.; FAÚNDES, A.; HARDY, E. E.; SIMÕES, I. R.; OSIS, M. J. D.; SOUZA, T. R. \& MORAES, T. M., 1990. Avaliação da assistência ginecológica no Estado de São Paulo. Revista de Ginecologia e Obstetrícia, 1:7-21.

POPULATION REPORTS, 1987. Os homens - novo enfoque para os programas de planejamento familiar. Population Reports, Série J, 33:1-36.

PINTO, I. A., 1987. Planejamento familiar. Generalidades, aspectos demográficos, políticos e sociais. In: Tratado de Ginecologia (H. Halbe, ed.), vol. 1, pp. 309-407. São Paulo: Editora Rocca.

ROGOW, D., 1992. Salud reproductiva masculina. Revista de Salud de las Mujeres Latinoamericanas y del Caribe, 2:3-56.

SAKAMOTO, C. P. M.; FREIRE, H. S. \& MORRIS, L., 1989. O Homem e a Vasectomia na Cidade de São Paulo. Um Estudo de Conhecimento, Atitudes e Comportamento Fase II. São Paulo: Centro Materno-Infantil. 